\title{
Learning by gaming - evaluation of an online game for children
}

\author{
Lisa J. Lazareck, Member, IEEE, David Farrell, Patty Kostkova, Member, IEEE, \\ Donna M. Lecky, Cliodna A. M. McNulty, Dasun Weerasinghe
}

\begin{abstract}
Playing computer games is widely popular among children and teenagers as an entertainment activity; meanwhile, playing computer games also provides a learning opportunity. For example, the rules of the game have to be learned by the player in order to improve his/her performance. Based on that principle, the City eHealth Research Centre (CeRC) developed a web game for 13-15 year olds, whereby the player becomes an investigator who attends the scene of an incident that involves microbes. There are four missions in total, each involving a mystery that the player needs to solve and learning objectives that need to be taught - such as antibiotic resistance and the importance of hygiene. This paper presents the results from a game evaluation that took place between July of 2009, in four UK schools (Glasgow, Gloucester, London), with 129 students; whereby $98 \%$ of the students commented positively about playing the game. Subsequently, CeRC has improved the game and developed an interactive educational games portal (www.edugames4all.com) for different age groups of web game enthusiasts.
\end{abstract}

\section{INTRODUCTION}

Oom omputer games are part of most children"s lives in the developed world $[1,2]$. Playing computer games provides a learning experience for both the novice and

the expert player, such that the rules of the game can be learned and the playeres performance can be improved. In addition to the learning that happens whilst game playing, the popularity and "motivational power of games" has lead to the development of games as a tool for education [3]. Research into educational gaming is said to significantly exist in the disciplines of medical education and business management, according to Wideman et al. [4]. Most recently, the City eHealth Research Centre (CeRC) at the City University London (UK), has developed such educational computer games to improve young people ee $^{\text {s }}$ understanding of the importance of hand and respiratory hygiene and responsible antibiotic use; and to teach school syllabus concepts such as microbes, the spread and prevention of infection, antibiotic use and antibiotic resistance. These games act as a standalone companion to the

Manuscript received April 23, 2010. This work was supported by the Directorate General for Health and Consumer Affairs (DG SANCO) of the European Commission (2005211).

L. J. Lazareck, D. Farrell, P. Kostkova, D. Weerasinghe are with the City eHealth Research Centre (CeRC), School of Community and Health Sciences (SC\&HS), City University London, Northampton Square, London, EC1V 0HB (phone: +44 207040 4136, fax: +44 207040 8795, email: lisa.lazareck@city.ac.uk).

D. Lecky and C. A. M. McNulty are with the Health Protection Agency (HPA), Primary Care Unit, Microbiology Department, Gloucestershire Royal Hospital, Great Western Road, Gloucester, GL1 3NN. teaching packs developed for the e-Bug Project: a European wide teaching resource for junior and senior school children.

The e-Bug Project was created to complement existing national hygiene and antibiotic educational campaigns, with 17 countries involved covering $62 \%$ of the European population (www.e-bug.eu). The Project was funded by DG SANCO, the Directorate General for Health and Consumer Affairs. The e-Bug learning packs were distributed across each associated country, with links to each partner country ${ }^{\text {ee }}$ s curriculum. Alongside the paper learning packs, the Project piloted the new and popular medium of (educational) gaming to teach children a set of learning outcomes or LOs (as agreed by the scientific partners working on the Project); i.e., for children to explore such areas as hand washing, respiratory hygiene, and antibiotic resistance. Two web browser-based games were developed, having been designed for two target audiences: the Junior Game for junior school pupils (9-12 years), and the Senior Game for senior school pupils (13-15 years of age). Playable at home or within the classroom, these games offer an engaging and entertaining way for pupils to study microbiology at their age-appropriate level. Each game was developed using user-centered design techniques such that each target audience was placed at the centre of their game design. By frequently engaging with the play-testers, it was possible to iteratively develop the games to ensure player satisfaction. Although a game may be designed on paper, the experience of actual playing is impossible to assess without testing the game with target users. Throughout development, there were frequent developmental evaluation play-tests, focus groups and observation studies that were used to test new features and to ensure that the games would be enjoyed by the target user (see [5], for example). Each game uses a different pedagogy to teach its content and provides a unique playing experience. The final versions of the games have been translated into 11 European languages, whereby support, translation, and modification requests were provided by the eBug Partners throughout the course of the project.

In this paper, we present an overview of the e-Bug Project, with an emphasis on the Senior Game - including its pedagogical design, development, and a discussion on the future potential of its reusable game platform.

\section{SENIOR GAME}

The Senior Game was designed for 13-15 year olds, whereby the player becomes an "e-Bug Investigator" who attends the scene of an incident that involves microbes. There are four missions in total, each involving a mystery that the player needs to solve. The overall mystery of each 
mission is sub-divided into multiple problems that must first be addressed, leading the player to their overall conclusion. The player must find evidence to support and negate their own ideas, in addition to those presented by the characters in the game. In the process of solving the mysteries, the player learns about microbes. In „Mission 0: Training, " the playeris introduced to the game concept and design, and is shown all of the pertinent features needed to complete each puzzle within the missions. In ,Mission 1: Bad Bacteria at the Barbeque Bash," actor Hugh Gaego has fallen ill after winning the award for best actor at the movie awards. His security guard Heracles Samson thinks that Hugh was deliberately poisoned by the chef who is the brother of another actor who failed to win any award. The player investigates a kitchen and bathroom for evidence of poor hygiene alongside Alyx (e-Bug Investigator) and is able to identify the cause of infection as well as discover some of the reasons why hygiene is important, which s/he reports to Big C (the Boss). Fig.1 illustrates the main characters utilized in Mission 1. Furthermore, Fig.2 illustrates the toilet under inspection by the investigators. Hugh Gaego ${ }^{\text {ee }}$ s toilet is depicted with and without the special ,microbe-vision ${ }^{\text {ee }}$ functionality, which is used to illuminate the various microbes present. In „Mission 2: When Bugs go Wild, "there are reports of a disease across Europe that can't be cured by normal means; i.e., even the most powerful antibiotics are ineffective. For some reason, the disease is prevalent in some areas of Europe more than others. The player visits the lab of a leading microbiologist as well as a doctor"s office in an area suffering from the disease. By discovering a link between inappropriate antibiotic use and cases of the disease, the player achieves an understanding of antibiotic resistance. In „Mission 3: Gambling Never Pays, ${ }^{\text {"e }}$ the player is called to investigate a situation where a football team has suddenly became ill and foul play is suspected. Despite the initial appearances of a football coach who is motivated to cheat, the player discovers that there may be a microbial cause of the problem. The player learns the importance of hygiene in handling water bottles, the implications of not finishing a course of antibiotics, and the side effects of using someone else ${ }^{e c}$ s antibiotics.

\section{A. Problem-Based Learning}

Adventure games are largely story driven, and because stories require a lot of text and interactivity, the Problem Based Learning (PBL) pedagogy utilized. PBL is "any learning environment in which the problem drives the learning; i.e., before students learn some knowledge, they are given a problem" [6]. By posing a problem, students are able to contextualize new knowledge instead of simply learning stand-alone facts. PBL encourages independent learning with minimal intervention from a teacher. Used extensively in medical training in higher education, PBL can be adapted to teach a wide range of LOs, making it suitable for the e-Bug Project [7]. PBL has been shown to be an effective approach to games-based learning, whereby Chen et al. found a statistically significant superiority for medical interns educated using PBL (rather than without PBL) in such areas as professional knowledge and clinical techniques [8].

There are five stages of PBL, according to the Queen ${ }^{\text {ee }}$ University definition for students [9]. Stage 1: the facilitator provides Topic Introduction, and highlights why the topic is relevant to the student. Stage 2: students write the Problem Statement in their own words, and care is taken to ensure that they correctly understand the problem. Stage 3: students Hypothesize potential solutions to the problem and identify one to pursue. Stage 4: students carry out Research, and seek information that confirms or refutes their hypothesis. Stage 5: students consider whether their hypothesis is confirmed through evidence - and if so, they Present their Result or findings with an emphasis on methodology, rather than the final answer. If the hypothesis is proved incorrect, students return to Stage 3 and identify a new hypothesis to investigate. For example, in Mission 1 - Hugh Gaego falls ill (Stage 1) and after the situation is explained (Stage 2), the player believes that he may have been food poisoned (Stage 3). Upon visiting the kitchen, the player gathers evidence for testing like the chopping board (Stage 4), whereby any microbes found may match those found from within Hugh (Stage 5). The development of the Senior Game is further described in [10].

\section{B. Technology Behind the Story-Game Engine}

In order to ensure that the Senior Game is accessible to as wide an audience as possible, the game was developed using Flash ActionScript CS2, targeting the Flash 8 web browser plug-in [11]. This plug-in has a wide penetration $(95 \%$ of internet enabled machines [12]) and, as such, does not typically require the target user to have administrative rights to install new software to their machine.

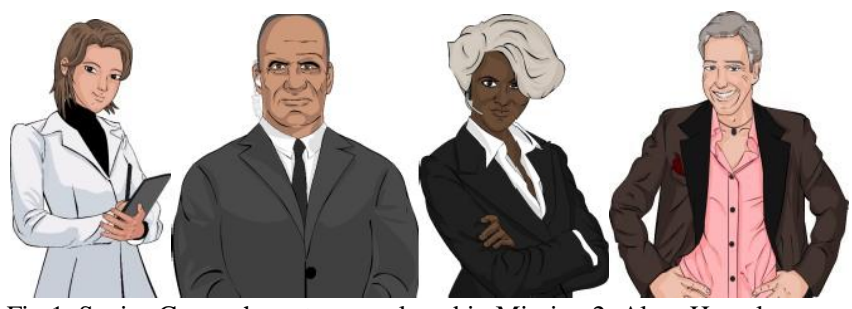

Fig.1. Senior Game characters employed in Mission 2: Alyx, Heracles Samson, Big C, Hugh Gaego.

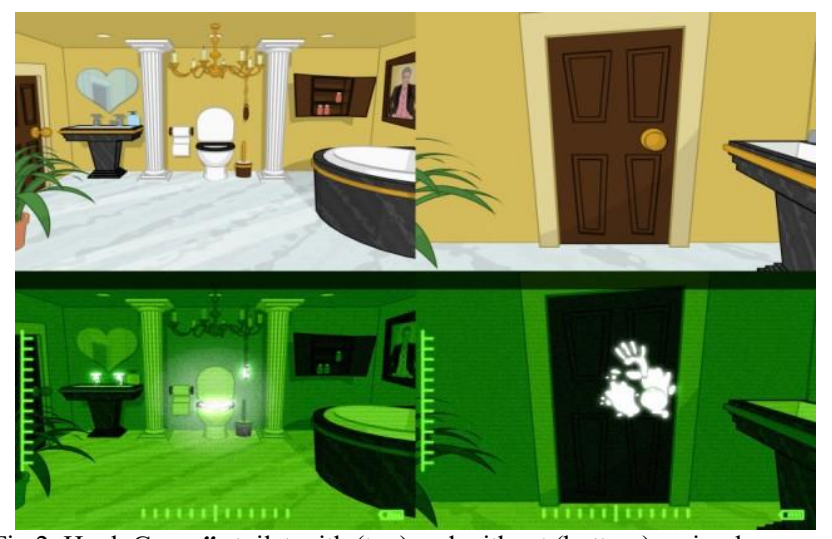

Fig.2. Hugh Gaego"s toilet with (top) and without (bottom) „microbevision." 
The Story-Game Engine is built such that it can power any story without having to make changes to the underlying technology; i.e., the story-game is data driven. This means that when the story-game is accessed, it loads text files which define the characters, rooms, objects, graphics (or art assets), and lines of dialogue used in the playing scenario. All of the puzzle logic is also loaded from these text files; i.e., if a change is required to the story-game logic, it is not necessary to edit the coded software, only the contents of the text files (and a subsequent re-run of the story-game).

The text files are created via an Excel spreadsheet (.xls). This spreadsheet has a format that guides the designer including several tabs (or new spreadsheet pages) for each element of the design. For example, if the designer wants to write a new character into the game, s/he accesses the „Character"e tab and fills out the requisite fields. Similarly, if the designer wants to edit or add dialogue, s/he accesses the „Conversations ${ }^{\text {ee }}$ tab and edits accordingly for the character of interest, etc. Each spreadsheet is saved as its own Comma Separated Value (.csv) file for the story-game to load during compilation. The web page that embeds the story-game can then pass variables to the game to define which scenario is loaded. In this way, the same code can be used in multiple web pages and can show a different scenario each time.

\section{Facilitating Multi-Lingual Games}

Because the story-game is data driven, it is possible to replace the text files with their translated equivalents that will be accessed by the story-game during compilation. To facilitate this process for the Senior Game, at first, the English language version of each scenario was written and tested. Next, the finalized spreadsheet was sent to each partner country for translation. The puzzle logic remains as originally designed; therefore, although the text changes language, it is trivial to switch language files (.xls) based on user preference. For example, the e-Bug Detective Games are implemented in 11 European languages (English, Belgian-French, Flemish, Czech, Danish, French, Greek, Italian, Polish, Portuguese, and Spanish), with the European Centre of Disease Prevention and Control (ECDC) already in agreement to fund further translations into the other remaining major European languages.

\section{Evaluation OF THE SENIOR GAME}

Throughout the development of the Senior Game, focus groups and observational studies at secondary schools were performed and evaluated in order to aid game focus and improvement. In June of 2009, the game ees ultimate developmental evaluation transpired with 12 pupils ( 8 female, 4 male) from one school in London. The results of this evaluation were utilized to iteratively improve the game performance before its evaluation in July. For example, during this developmental evaluation, 9 out of 12 pupils had difficulty actually playing the game as it either froze or crashed entirely. Subsequently, the game developer fixed these problems before proceeding further with testing.
The Senior Game evaluation took place in July of 2009, in four UK schools (Glasgow, Gloucester, London). (One school was visited twice during the months of June and July.) The 129 pupils were selected as a convenience sample, all falling within the target age demographic for the Senior Game, with an average of 13 years. The total number of pupils encountered per school visit included: 6 ( 2 female, 4 male), 52 (22 female, 29 male, 1 unspecified), 57 (26 female,

31 male), and 14 (14 female, 0 male). All of the pupils played Mission 2 (only, as it was most suitable due to its duration and complexity) of the Senior Game in-school and over the internet. (Missions 1 and 3 had not been fully completed at the time.) Plus, of the 129 pupils in total, 116 had not played the game before; 11 had played; and 2 did not specify. The following questions were asked of each pupil in a paper survey: What did you like about the game?, What did you dislike about the game?, What did you think of the story?, What did you think of the Characters?, If you could only change one thing about the game, what would it be?, Is this a game that you would play only in school or would you play it in your own free time?, Play more than once?, Recommend to friends?, Do you have any other comments about the game?. The pupils could provide a variety of comments, including yes/no answers.

\section{A. Results \& Discussion}

Overall, the July evaluation revealed that $98 \%$ of the pupils had commented positively about the Senior Game. Some interesting remarks include: I thought it was interesting and I taught me how microbes are harmful; It was a good story for people of our age group but it was a bit too complicated; The story is very good because you can learn something from it...it's about science; It's quite a strong story and the investigating is quite cool; I liked the interesting storyline and the amount of interactivity used. The graphics were fairly good. The whole game overall was still good and exciting.

However, there were negative comments as well. There were comments about the difficulty of the game and the lack of any formal instruction. In order of school visits: $50 \%, 54 \%$, $42 \%$, and $29 \%$ of the pupils found it frustrating that they could not easily operate the game without preliminary instruction. As a result, the game developer created a training portion within the Senior Game; i.e., Mission 0, whereby the player is taught how to maneuver through the game one step at a time. Also, game play hints and tips were incorporated into each of the Missions, as well as a Help File (in .pdf form) that could be called upon during game play. Next, there were a few detrimental comments about the game freezing during play. In order of school visits: $0 \%, 2 \%, 5 \%$, and $7 \%$ of the pupils encountered this difficulty; however, this may have been due to the slow downloading speeds at the schools visited.

Table I shows the response tally to the following questions: Play more than once?, Recommend to friends?. Out of the 129 pupils, $46 \%$ would play game more than once, whereas $22 \%$ would not, $24 \%$ were not certain, and $8 \%$ did not answer the question. Similarly, $43 \%$ of the pupils would 
recommend the game to friends, $16 \%$ would not, $33 \%$ were not certain, and $8 \%$ did not answer the question. As a result of this evaluation, the Senior Game was revamped in August 2009, and then finalized in December 2010.

TABLE I

Response tally with overall percentage of the total pupils (129) provided.

\begin{tabular}{c|cccc|cccc}
\hline & \multicolumn{3}{c|}{ Play more than once? } & \multicolumn{3}{c}{ Recommend to friends? } \\
\hline Pupils & Yes & No & $\begin{array}{c}\text { Not } \\
\text { Sure }\end{array}$ & $*$ & Yes & No & $\begin{array}{c}\text { Not } \\
\text { Sure }\end{array}$ & $*$ \\
\hline 6 & 3 & 0 & 3 & 0 & 3 & 0 & 3 & 0 \\
52 & 22 & 11 & 17 & 2 & 20 & 9 & 21 & 2 \\
57 & 24 & 14 & 10 & 9 & 21 & 10 & 17 & 9 \\
14 & 10 & 3 & 1 & 0 & 11 & 1 & 2 & 0 \\
\hline Total: 129 & 59 & 28 & 31 & 11 & 55 & 20 & 43 & 11 \\
\hline$\%$ of Total & $46 \%$ & $22 \%$ & $24 \%$ & $8 \%$ & $43 \%$ & $16 \%$ & $33 \%$ & $8 \%$ \\
\hline
\end{tabular}

* No response provided.

\section{FUTURE WORK FOR THE STORY-GAME ENGINE}

A quantitative evaluation of the finalized Senior Game (post-December 2010) is currently underway. A successful outcome for the Senior Game evaluation will be defined as the acquisition of knowledge of the pre-defined set of LOs through game play or game engagement. It is also important to ascertain the players ${ }^{\text {ee }}$ attitude towards the Senior Game itself; i.e., do they enjoy the actual game playing and entertainment value of the game? Additional evaluation is also ongoing to ascertain how player opinion differs per country/language/culture, which will be accomplished via collaboration with the e-Bug Partners.

In addition to the existing e-Bug games for school children, CeRC is investigating the possibilities of using similar games to convey messages to particular academic and/or industrial communities. For example, games could be further utilized for adult education in the medical context - such as teaching about gender issues, sexually transmitted infections, infection control for hospital staff, etc. For example, in order to deliver a new game LO, a designer must create a story-based scenario which has a flaw or problem to solve, whilst simultaneously considering the clues, evidence, misinformation, and hints provided. Similarly to the example provided for the Senior Game (harmful microbes causing illness), any LO is possible to implement within the story-game as long as a logic puzzle can be formulated accordingly. For instance, for an agricultural-themed game, several LOs about providing fair trade principles to propose a sustainable development solution for a small town can be incorporated into a story-game investigating the impact of under-priced supermarket products on local farming communities. The cost of re-using this (open-sourced) framework is minimal, and the platform enables the efficient development of new story-based educational games with new learning objectives along with the ability to support translation into any language.

Lastly, CeRC has created www.edugames4all.com: an interactive educational website that hosts, evaluates, and evolves internet games and training simulators for audiences of all generations in a variety of disciplines. Both the Junior and Senior Games, and their source files and illustrations, are available from within this website.

\section{ACKNOWLEDGMENT}

We acknowledge the members of the e-Bug working group (which also include the authors): Niels Adriaenssens, Natasha Barbouni, Jiří Beneš, Antonio Brito Avo, Carla Rodriguez Caballero, José Campos, Guiseppe Cornaglia, Charles Davis, Stijn De Corte, Ed de Quincey, Pierre Dellamonica, Dimitra Gennimata, Herman Goossens, Pawel Grzesiowski, Tereza Herotová, Jette Holt, Gawesh Jawaheer, Raffaella Koncan, Jenny Kremastinou, Koula Merakou, Marianne Noer, Monika Nowakowska, Anna Olczak- Pienkowska, Pia Touboul, Julius Weinberg. Also, thanks to digital artists Nancy Lai and Sandy Beveridge, who created the project artwork. Lastly, we acknowledge and thank the schools and children that took part in the evaluation as well as those who played the games online and continue to do so.

\section{REFERENCES}

[1] S. Anderson, C.D. Economos, A. Must. "Active play and screen time in US children aged 4 to 11 years in relation to sociodemographic and weight status characteristics: a nationally representative crosssectional analysis," BMC Public Health, vol. 8, pp. 366, 2008.

[2] J.I.I. Bengoechea. "Videogames and education: a first empirical research in the Basque Country," in 2009 Proc. 3rd European Conference on Games Based Learning, pp. 195 - 201.

[3] J. Kirriemuir, A. McFarlane. (2004, accessed) Literature review in games and learning, Bristol: FutureLab. Available: http://www.futurelab.org.uk/resources/documents/lit_reviews/Games_Review.pdf

[4] H.H. Wideman, R.D. Owston, C. Brown, A. Kushniruk, F. Ho, K.C. Pitts "Unpacking the potential of educational gaming: A new tool for gaming research," Simulation and Gaming, vol. 38, no. 1, pp. 10-30, 2007.

[5] D. Farrell, P. Kostkova, J. Weinberg, L. Lazareck, D. Weerasinghe, C.A.M. McNulty. "Can computer games wash your hands? An evaluation of the e-Bug junior game," $J$. of Antimicrobial Chemotherapy, in-press, Summer 2010.

[6] McMaster Problem Based Learning Program (2009, accessed). Available: http://chemeng.mcmaster.ca/pbl/PBL.HTM

[7] Annetta, L A. Cook, M, Schultz, M. "Video Games: A Vehicle for Problem Based Learning." Technology-Enhanced Education Part II Springer. Netherlands. 2009.

[8] Y-C Chen, J-T Fang, J-D Lin, W-J.Cherng. "Evaluation of problembased learning Education after clerkship at the Chang Gung University School of Medicine," Chang Gung Medical J., vol. 25, no. 11, 2007.

[9] Queens University Problem Based Learning (2009, accessed). Available: http://meds.queensu.ca/pbl/pbl in_practice/pbl_process

[10] D. Farrell, P. Kostkova, L. Lazareck, D. Weerasinghe. "Developing web games to teach microbiology," J. of Antimicrobial Chemotherapy, in-press, Summer 2010.

[11] D. Farrell, P. Kostkova, L. Lazareck, D. Weerasinghe. "CeRC StoryGame Engine: An open source technology to power story based investigation games," in 2010 Proc. Med-e-Tel Conf.: International eHealth Telemedicine and Health ICT Forum for Education, Networking and Business, in-press.

[12] 2007 Millward Brown survey carried out for Adobe.com (January 2010 accessed).Available:http://www.adobe.com/products/player_census/flashplayer /version_penetration.html 